\title{
Opciones para reducir la pobreza rural en América Latina y el Caribe
}

\section{Rubén G. Echeverría}

Departamento del

Desarrollo Sostenible,

Banco Interamericano

de Desarrollo

RUBENECHE@aol.com
Aunque la mayoría de la población total y de la población pobre en América Latina y el Caribe se encuentra en los centros urbanos, en términos relativos la pobreza sigue siendo un fenómeno rural en la región. La incidencia de la pobreza y de la pobreza extrema es mucho mayor en las zonas rurales que en las urbanas. Sin ir más allá, en 1997 más de la mitad de los hogares rurales vivía en la pobreza y cerca de un tercio en situación de pobreza extrema. Además, la difícil situación económica de la mayoría de los países de la región en los últimos dos años seguramente ha deteriorado esas cifras. A los pobres de las zonas rurales de la región se les plantean por lo menos tres problemas básicos: i) nutrición insuficiente, mala salud y servicios educativos deficientes; ii) escasas oportunidades de empleo productivo en la agricultura y en actividades no agrícolas; y iii) bajo grado de organización para promover efectivamente los intereses rurales. El número y la variedad de circunstancias que causan la pobreza rural, así como la heterogeneidad de condiciones en que se presenta en los distintos países y regiones dificultan la búsqueda de soluciones efectivas y eficientes para elevar el bienestar de la población rural. Este artículo tiene como objetivo destacar varias opciones de acción para reducir la pobreza rural en la región. Para este fin, se centra en tres tipos de posibilidades de acción importantes y complementarias para generar o elevar los ingresos de los pobres de las zonas rurales: uno que se basa en el crecimiento del sector agrícola; otro que apunta al uso sostenible y la conservación de los recursos naturales, y el último basado en la importancia creciente de las actividades económicas rurales que se desarrollan fuera del predio agrícola. Hay al menos otras dos posibilidades de reducción de la pobreza rural: la tradicional migración a las zonas urbanas y la asistencia destinada a las personas que necesitan transferencias de ingresos para superar el umbral de pobreza o para tener un mínimo acceso a las redes de seguridad social. 


\section{I}

\section{Introducción}

Aunque el $74 \%$ de la población y el $62 \%$ de los habitantes pobres de América Latina y el Caribe se encuentran en centros urbanos, en términos relativos la pobreza sigue siendo un fenómeno rural en la región. La incidencia de la pobreza y la extrema pobreza es mayor en las zonas rurales que en las urbanas. Además, el ingreso medio rural es bastante más bajo que el urbano. En 1997 alrededor del 75\% o más de la población rural de Bolivia, Ecuador, Guatemala, Haití, Honduras y Nicaragua se encontraba bajo el umbral de pobreza. En Brasil, México y Colombia (países que registran las mayores poblaciones rurales pobres con aproximadamente 20,15 y 6.5 millones respectivamente), entre el 56 y el $62 \%$ de la población rural se encontraba bajo el umbral de pobreza (CEPAL, 1999). El número y diversidad de circunstancias que causan la pobreza rural hacen que sea difícil concebir desde el punto de vista económico soluciones eficaces para elevar el bienestar de la población rural. El objetivo del presente artículo es señalar varias opciones para reducir la pobreza rural en la región.

\section{II \\ La magnitud y heterogeneidad de la pobreza rural}

Más de una cuarta parte de la población de América Latina vive con menos de un dólar por día. Las mayores concentraciones de pobreza se hallan en Centroamérica, la región andina y el Nordeste brasileño, donde un $60 \%$ de los habitantes vive por debajo del umbral de la pobreza. Entre 1994 y 1997, el porcentaje de hogares rurales en situación de pobreza bajó en sólo dos puntos porcentuales: de 56 a 54\%; por su parte, la proporción de la población rural en situación de extrema pobreza disminuyó de 34 a $31 \%$ en el mismo período (cuadro 1). Pese a este modesto avance, la cifra correspondiente a la pobreza rural en 1995 (54\% para la región, en términos relativos) es parecida a la de 1980, mientras que la de pobreza extrema es más alta $(31 \%$ frente a $28 \%)$.

Desde comienzos del decenio de 1990, los pobres de las zonas urbanas (principalmente mujeres jefes de hogar, padres con educación formal escasa o nula y jóvenes desempleados) por primera vez han llegado a ser más numerosos que los de las zonas rurales; los

El autor agradece las sugerencias de un juez anónimo que colabora con la Revista de la CEPAL y los comentarios de Martine Dirven. Las opiniones expresadas en este artículo no coinciden necesariamente con las de la organización a la que el autor pertenece.

\begin{tabular}{|c|c|c|c|c|c|c|}
\hline \multirow{3}{*}{ CUADRO 1} & \multicolumn{6}{|c|}{$\begin{array}{l}\text { América Latina: Hogares en situación de } \\
\text { pobreza y extrema pobreza, 1980-1997 } \\
\text { (Porcentajes) }\end{array}$} \\
\hline & \multicolumn{3}{|c|}{ Pobreza } & \multicolumn{3}{|c|}{ Extrema pobreza } \\
\hline & Total & Urbana & Rural & Total & Urbana & Rural \\
\hline 1980 & 35 & 25 & 54 & 15 & 9 & 28 \\
\hline 1990 & 41 & 35 & 58 & 18 & 12 & 34 \\
\hline 1994 & 38 & 32 & 56 & 16 & 11 & 34 \\
\hline 1997 & 36 & 30 & 54 & 15 & 10 & 31 \\
\hline
\end{tabular}

Fuente: CEPAL (1999).

primeros se estiman actualmente en más de 126 millones, mientras que los últimos suman 78 millones (cuadro 2). Sin embargo, hay más habitantes rurales que urbanos en situación de extrema pobreza (47 millones en comparación con 42.7 millones), aunque un alto porcentaje de los pobres que viven en zonas urbanas son recién llegados desde las rurales. El leve descenso en la proporción de hogares en situación de pobreza y de extrema pobreza en las zonas rurales en el decenio de 1990 (de 58 y 34\% a 54 y 31\%, respectivamente) no ha impedido que aumente el número absoluto de personas afectadas (en comparación con el decenio de 1980). Además, la difícil situación eco- 
nómica en la mayor parte de la región en 1998 y 1999 podría haber hecho subir en vez de bajar estas cifras ya de por sí alarmantes.

La pobreza rural es más aguda que la urbana y parece más difícil de superar. En muchos países de la región los pobres en áreas rurales todavía no se benefician del crecimiento económico de los últimos tiempos. La distribución desigual de la tierra y las distorsiones del mercado de servicios agrícolas han contribuido a perpetuar la pobreza rural. Aunque la incidencia de ésta varía grandemente entre los países y dentro de los territorios nacionales, su magnitud y difusión son tales que resulta imprescindible conceptualizar y ejecutar programas eficaces para reducirla. En los países más urbanizados comienza a descender la migración a las ciudades, pero siguen prevaleciendo altas tasas de migración hacia zonas urbanas en aquellos países en que la población rural representa más de $25 \%$ de la población total. Es imperativo crear las condiciones para que la población rural pueda alcanzar niveles de vida (económicos, políticos, sociales y culturales) parecidos a los que tienen los habitantes urbanos.

Paradójicamente, aunque los sectores agrícolas de la mayoría de los países registraron un crecimiento considerable en el decenio de 1990, no están bajando los índices de marginalización y pobreza rural. Es evidente también que el crecimiento económico ha contribuido por lo menos a frenar el aumento de la pobreza rural, gracias a una mayor demanda de mano

\begin{tabular}{lrrrrrr} 
CUADRO 2 & \multicolumn{3}{c}{$\begin{array}{l}\text { América Latina: Personas en situación de } \\
\text { pobreza y extrema pobreza, 1980-1997 } \\
\text { (Millones) }\end{array}$} \\
\hline \multicolumn{3}{c}{ Pobreza } & \multicolumn{4}{c}{ Extrema pobreza } \\
\cline { 2 - 7 } & Total & Urbana & Rural & Total & Urbana & Rural \\
\hline 1980 & 135,9 & 62,9 & 73,0 & 62,4 & 22,5 & 39,9 \\
1990 & 200,2 & 121,7 & 78,5 & 93,4 & 45,0 & 48,4 \\
1994 & 201,5 & 125,9 & 75,6 & 91,6 & 44,3 & 47,4 \\
1997 & 204,0 & 125,8 & 78,2 & 89,8 & 42,7 & 47,0 \\
\hline
\end{tabular}

Fuente: CEPAL (1999). de obra y las consiguientes migraciones, lo que prueba que parte de la solución está fuera del sector agrícola propiamente dicho. En consecuencia, el ingreso no agrícola ha llegado a representar una proporción mayor del ingreso rural total: alcanza niveles de $68 \%$ en Haití, 59\% en Costa Rica y 51\% en Argentina y se acerca al $50 \%$ en un promedio de 13 países (Reardon y Berdegué, 1999). Sin embargo, el crecimiento económico no ha sido suficiente en todos los casos, lo que indica que las condiciones de pobreza extrema en las zonas rurales sólo podrán mejorarse con programas destinados específicamente a tal efecto.

¿Quiénes son los pobres de las zonas rurales? Aunque no se dispone de datos recientes, al finalizar el decenio de 1980 los pequeños agricultores representaban la mayor proporción (más del 60\%), mientras que los habitantes sin tierra y los grupos indígenas y otros constituían, respectivamente, el 30 y el 4\% (FAO, 1988). Según varias investigaciones, por lo menos un tercio de los pequeños productores mencionados eran agricultores de subsistencia, con escasas oportunidades de superar la pobreza por medio de la agricultura. Esto indica que entre el 40 y el $50 \%$ de los pobres de las zonas rurales pueden tener un acceso muy limitado a recursos productivos que les permitan obtener suficientes ingresos de la producción agrícola misma. Además, la población rural con menor acceso a los recursos agrícolas crece con más rapidez que la que tiene mayor acceso.

La población rural pobre de la región enfrenta por lo menos tres problemas básicos: i) nutrición insuficiente, mala salud y servicios educativos deficientes; ii) escasas oportunidades de empleo productivo en la agricultura y en actividades no agrícolas, y iii) grado insuficiente de organización para promover efectivamente los intereses rurales. Las opciones para reducir la pobreza rural que se presentan en este artículo se basan en estos desafíos, así como en la heterogeneidad de las condiciones de pobreza rural entre los distintos países y regiones y dentro de ellos. Antes de analizar esas opciones conviene pasar revista al nuevo contexto regional para el desarrollo rural y a algunas lecciones que se han aprendido de la experiencia. 


\section{III}

\section{El nuevo contexto regional para el desarrollo rural}

Varias tendencias importantes influyen en la evolución de las zonas rurales de la región: la mayor integración económica y la liberalización del comercio; la nueva concepción del papel del Estado, y la mayor conciencia de los problemas vinculados con el medio ambiente, el género y las etnias (Echeverría, 1998).

\section{Mayor liberalización, integración y apertura comercial}

Los tratados bilaterales, el Tratado de Libre Comercio de América del Norte y el Mercosur, los acuerdos con la Comunidad Europea y los países del foro de Cooperación Económica del Asia-Pacífico (APEC), así como las deliberaciones con miras a la creación del Tratado de Libre Comercio para las Américas, han contribuido a una mayor apertura de los mercados y a una integración más estrecha del sector agroalimentario y agroindustrial de la región. Las nuevas oportunidades del mercado inducen a los productores a dedicarse a las exportaciones y permiten a la vez la importación de productos agrícolas desde fuera de la región. El deseo de mejorar la competitividad se traduce en un mayor esfuerzo por elevar la productividad y la calidad, lo que lleva a una mayor concentración o diversificación en el comercio y la agroindustria. Las iniciativas en este campo se han visto acompañadas en muchos casos (Argentina, Brasil, México y Chile) por la formación de empresas conjuntas con inversionistas locales en sectores agrícolas y no agrícolas, así como de fusiones y una mayor concentración entre las empresas nacionales. La mayor escala de producción (sobre todo de cereales, carne, oleaginosas y azúcar, pero también de ciertas frutas y productos industriales) junto con la mecanización, computarización e internacionalización de las actividades agrícolas afectan el empleo rural y los mercados de tierras. Ante los desafíos de la libre competencia, los minifundistas que explotan tierras marginales y tienen acceso limitado a la educación, el progreso tecnológico, las comunicaciones y la información se encuentran en desventaja. $\mathrm{Su}$ situación en los mercados le da poca opción a este grupo para competir en forma eficaz y sostenida en este nuevo contexto.

\section{Un nuevo papel para el Estado}

La liberalización mediante la privatización y el ajuste estructural para reducir el déficit fiscal se han traducido no sólo en una menor intervención del Estado en los asuntos rurales sino también en el desmantelamiento de las organizaciones que tradicionalmente se ocupaban del sector agrícola, con lo cual se ha dificultado el desarrollo rural hasta que esas organizaciones puedan ser reemplazadas por la sociedad civil y el sector privado, sobre todo en el plano local (Piñeiro, Martínez Nogueira, Trigo, Torres, Manciana y Echeverría, 1999). La reducción o retiro de los servicios del sector público ha sido notable especialmente en algunas áreas que afectan a la vida rural como el gasto social (en educación, en salud), el sistema financiero, la infraestructura y servicios agrícolas claves como los de tecnología. En estas áreas, cuanto mayor es la demanda de inversión en capital humano, crédito, comunicaciones e investigación para atender la necesidad de mejorar la competitividad rural, menores son las oportunidades de acceso de la población rural a esos servicios. $^{1}$

Los países que se proponen remediar esta deficiencia y se empeñan en invertir en un crecimiento económico con equidad social suelen tropezar con dificultades para desarrollar programas eficaces que permitan acabar con el estatismo tradicional y la burocracia excesiva de sus administraciones, promoviendo en cambio la participación activa de las comunidades locales y el sector privado y utilizando los mecanismos del mercado en la tentativa de reducir la pobreza. Pese a las dificultades, avanza en la región la economía de mercado, la incorporación de nuevos agentes como las organizaciones no gubernamentales, y la búsqueda de un nuevo equilibrio entre los papeles de los sectores

\footnotetext{
${ }^{1}$ Por otra parte, la liberalización de los mercados y los programas de ajuste han reducido en cierta medida el sesgo urbano surgido del proceso de industrialización impulsado por el Estado, que penalizaba a la agricultura como sector productivo mientras compensaba a los terratenientes con subsidios vinculados a la propiedad de la tierra (Binswanger y Deininger, 1997).
} 
público y privado y la sociedad civil en el desarrollo. Sin embargo, el avance en la transición de un Estado centralizado a un Estado con funciones normativas, junto con la participación más activa de los grupos no estatales en beneficio de los segmentos más pobres de la sociedad, es incipiente en el mejor de los casos y debe ser promovido con más fuerza.

\section{Mayor conciencia de los problemas del medio ambiente, el género y las etnias}

La gran mayoría de los pobres de áreas rurales vive en zonas de escaso potencial agrícola, incluidas áreas degradadas o semiáridas, terrenos propensos a la erosión o con suelos frágiles y pendientes inclinadas, en las estribaciones de los Andes, las cordilleras costeras de México y Centroamérica o las zonas áridas del Nordeste brasileño. Esta población depende para su subsistencia (agua, alimentos, energía e ingresos) de los recursos naturales; sin embargo, porque éstos son de mala calidad y escasos, muchas poblaciones no tienen más remedio que transgredir los límites de lo sostenible y agotar estos recursos. Pese a la abundancia de recursos naturales en la región, los problemas de la desertificación, la destrucción de tales recursos, el cambio climático y la pérdida de biodiversidad ejercen una creciente presión en la búsqueda de medios eficaces para lograr un desarrollo sostenible. Por esta razón, romper el círculo vicioso de la deforestación, la degradación de los recursos de agua y suelo y el aumento de la pobreza rural constituye uno de los elementos claves que configuran las nuevas estrategias rurales.

A los pueblos y gobiernos de la región les preocupa cada vez más la calidad del medio ambiente. Aunque se sigue favoreciendo al medio urbano en la asignación del gasto ambiental, aumenta el número de programas y proyectos de conservación destinados a proteger la tierra, los recursos hídricos y los bosques. La insistencia en el aprovechamiento sostenible de los recursos naturales no sólo guarda relación con la demanda ciudadana de una mejor calidad de vida sino que también brinda posibilidades para el desarrollo de las propias comunidades rurales. Del mismo modo, el uso de tecnologías y sistemas respetuosos del medio ambiente que aseguren el desarrollo sostenible de los recursos naturales también ofrece la posibilidad, limi- tada hasta ahora, de generar nuevos productos (orgánicos o verdes) así como de crear y adoptar nuevas tecnologías más al alcance del pequeño agricultor.

Aunque hay variaciones entre los países y pese al hecho de que el trabajo de la mujer a veces no aparece en las estadísticas nacionales, es posible que las mujeres en áreas rurales produzcan hasta un $50 \%$ del alimento consumido en la región. En muchos países son las mujeres las que administran los predios, recogen la leña y el agua y cuidan del ganado, mientras sus esposos e hijos mayores trabajan en la agricultura comercial o en los centros urbanos. La proporción de mujeres en la fuerza trabajadora rural subió del 21 al 25\% entre 1980 y 1995 y según las proyecciones llegará al 28\% de la población económicamente activa en el campo hacia el año 2010 (Dirven, 1997). El descenso de la importancia relativa de las actividades agrícolas (y sobre todo de la producción de autoconsumo), la expansión de los sectores del comercio y los servicios y la difusión del trabajo remunerado a las zonas rurales han facilitado la incorporación de la mujer en los mercados de trabajo y al mismo tiempo han hecho más visible su participación.

Históricamente, la mujer y las poblaciones indígenas no han tenido un acceso equitativo a la tierra, el crédito, el capital y los servicios de extensión, aunque estos grupos son precisamente los que más utilizan los recursos hídricos, edafológicos y forestales tanto para proveer a las necesidades básicas de sus familias como para obtener ingresos adicionales. Aunque las recientes reformas legislativas han contribuido mucho a remediar estos problemas, persiste una cierta discriminación. En muchos casos las comunidades indígenas viven en las zonas más frágiles (sobre todo en zonas aisladas de bajo potencial agrícola y escasas posibilidades de empleo no agrícola), a menudo contiguas a reservas naturales o dentro de ellas. En consecuencia, los planes de conservación para las zonas protegidas (como en el caso de cualesquiera otras tierras ocupadas por comunidades indígenas) tendrían mucho mejores posibilidades de éxito si incorporaran a estas comunidades en los programas de gestión ambiental, garantizando la integridad de sus territorios y mejorando su acceso a los servicios sociales. Además, es necesario concebir programas de desarrollo rural destinados específicamente a reducir la pobreza entre los grupos indígenas. 


\section{IV}

\section{Algunas lecciones aprendidas}

Hasta ahora no se ha elaborado ningún plan regional que aborde el problema de la pobreza rural en la región en toda su magnitud y persistencia, ni se han asignado sistemáticamente suficientes recursos a nivel nacional para remediarlo. Es más, hasta ahora la mayoría de los proyectos rurales han constituido actividades inconexas y discontinuas. En algunos casos, las políticas rurales han coexistido con políticas contradictorias como las de importación de productos agrícolas altamente subvencionados, sobrevaloración de los tipos de cambio, control de los precios de los productos básicos y fijación de altas tasas de interés, o bien han procurado compensar esas políticas. De las diversas experiencias en materia de reducción de la pobreza rural derivan las cuatro lecciones siguientes sobre lo que debe hacerse.

\section{Promover un marco normativo e institucional favorable}

En vista del papel vital que desempeñan el crecimiento económico y el crecimiento sectorial en la reducción de la pobreza rural, para el éxito del desarrollo rural es indispensable promover un marco macroeconómico y sectorial favorable. Contar con un marco basado en el mantenimiento de políticas estables y en la creación de un clima propicio es imprescindible para la ejecución eficiente y descentralizada de proyectos. Ello supone la existencia de programas sociales (redes de seguridad) para aquellos que no pueden satisfacer sus necesidades básicas. Por ejemplo, la asignación de fondos de inversión social a zonas afectadas por la pobreza debe hacerse extensiva a muchas zonas rurales. La experiencia de América Latina confirma que el crecimiento económico no basta para reducir la pobreza. Son factores igualmente decisivos el tipo de crecimiento y las estrategias destinadas a beneficiar al máximo a los pobres. El crecimiento económico ha contribuido a reducir la pobreza rural sobre todo merced a la migración. Además, el desarrollo agrícola también ha contribuido a reducir la pobreza en las zonas rurales. De ello se desprende que, aunque un crecimiento vigoroso de la agricultura es básico para el desarrollo rural, siguen necesitándose programas que promuevan expresamente dicho desarrollo (BID, 2000). La formu- lación de políticas macroeconómicas y sectoriales destinadas a superar la pobreza rural es un requisito esencial, pero no suficiente para lograr este objetivo. Además de estas condiciones, deberán cumplirse otros requisitos como los que se enumeran a continuación.

\section{Aumentar las inversiones en salud, educación e infraestructura}

Ha sido muy desigual el progreso en lo que se refiere a la extensión, el nivel y la calidad de los programas de salud y educación rurales. Para muchos países sigue siendo un objetivo lejano el logro de las condiciones necesarias para ofrecer a todos los niños de las zonas rurales la posibilidad de completar los seis años de enseñanza primaria. En unos pocos países el objetivo actual es brindar pleno acceso a los jóvenes de las zonas rurales a una enseñanza secundaria de cuatro años. ${ }^{2}$ En general, la calidad de la educación rural es inferior a la de los cursos y programas equivalentes de las zonas urbanas, debido a la distribución dispersa de la población, las bajas tasas de matriculación y la falta de incentivos educacionales en el medio rural. Además, en los planes de estudio no se toman en cuenta las necesidades concretas de los alumnos que viven en las zonas rurales y se tiende a discriminar contra la mujer, los pueblos indígenas y las personas de ascendencia africana tanto en el suministro de infraestructura y materiales como en el enfoque de los programas y las oportunidades de estudios avanzados. Es importante velar por que esos grupos no sólo aporten su trabajo sino que también se beneficien de los componentes generadores de ingresos de los programas de inversión social.

\footnotetext{
${ }^{2}$ Pese a la importancia reconocida de la educación, López y Valdés (1997) dan cuenta de un rendimiento sorprendentemente bajo de las inversiones en la educación rural en América Latina. Señalan que en promedio el aumento de la escolaridad en un año entre las familias rurales eleva los ingresos de los hogares en menos de veinte dólares por persona. Al parecer, la principal contribución de la educación rural reside en preparar a los habitantes de las zonas rurales para la migración a las zonas urbanas. Estos resultados ocultan la necesidad verdadera de una educación que aumente la competitividad en las zonas rurales y en concreto de una mayor inversión en esferas como la formación profesional de la juventud en el campo.
} 
En vista de la creciente marginalización de ciertas regiones, su falta de oportunidades de empleo y el hecho de que una población decreciente suele dificultar el suministro de servicios básicos al resto de los habitantes, se hace necesario efectuar algunos ajustes compensatorios entre el gasto social y las fuentes locales de ingreso. Un buen ejemplo es la relación entre la infraestructura de transporte y los servicios de educación y salud: en muchos casos construir un camino y transportar a los alumnos por autobús es la mejor solución para las localidades rurales aisladas, o establecer un internado en un pueblo cercano puede ser preferible a diseminar un número considerable de escuelas y maestros en el ámbito rural. Las inversiones en salud, educación e infraestructura destinadas a los sectores más desposeídos pueden emplearse para remediar algunas de las desigualdades de ingresos en las zonas rurales.

\section{Tomar en cuenta la heterogeneidad rural y la participación de los beneficiarios}

La diversidad de la población rural, los cambios del medio económico y las diferencias en las capacidades de aprendizaje entre los participantes en los proyectos en curso también entran en conflicto con las rigideces inherentes a la formulación y ejecución de la mayoría de los proyectos de desarrollo. Además, la falta de participación de los beneficiarios lleva a veces a la incorporación de componentes que no tienen demanda, a la imposibilidad de ajustar los proyectos según las posibilidades de asimilación de las comunidades y al carácter general insostenible de los programas de desarrollo rural. En general, los pobres de las zonas rurales carecen de capacidad de negociación y tienen poca influencia en los gobiernos o los organismos internacionales. Además, son pocos los profesionales capacitados para llevar a cabo tales programas y en general se desconoce el contexto económico, social y cultural en que éstos se inscriben.

Gran parte de las evaluaciones de los programas de desarrollo rural ponen de relieve la importancia de hacer participar a la población local de forma más directa y continua, desde la fase del diseño hasta la de ejecución de las actividades. Sin embargo, rara vez se consulta a las comunidades o participan éstas plenamente en tales programas. La experiencia de muchos proyectos pequeños indica que puede obtenerse un buen grado de éxito y cumplimiento de los objetivos allí donde las comunidades ya cuentan con un cierto nivel de organización o donde la estrategia de los pro- yectos asigna una proporción relativamente alta de los recursos a la capacitación y al desarrollo institucional. Podrían promoverse actividades de capacitación (que abarquen los aspectos del ordenamiento jurídico del país, la estructura orgánica y el proceso de decisión democrático y la gestión y administración de organizaciones y proyectos) a fin de fortalecer la capacidad organizativa básica para participar en actividades rurales. También debería capacitarse al personal de los organismos estatales encargados de supervisar los programas rurales a fin de facilitar la participación de sus beneficiarios.

Aunque existen centenares de grupos cívicos y/o no gubernamentales (asociaciones de agricultores, grupos indígenas, fundaciones, grupos ambientalistas y de derechos civiles, etc.), en su gran mayoría están mal organizados y carecen de capacidad técnica, lo que limita su posibilidad de participar en las actividades de desarrollo rural. Han resultado muy útiles las consultas con organizaciones no gubernamentales, fundaciones, empresas privadas, cooperativas, asociaciones de comercio, federaciones o comunidades indígenas, organismos nacionales o locales, órganos religiosos u otros grupos afines para determinar las prioridades entre varias demandas de las comunidades rurales que compiten entre sí y para inducir a los grupos que tienen alguna capacidad técnica a participar en la preparación y la supervisión de los proyectos.

\section{Mejorar la ejecución de los programas rurales}

La ejecución de los programas de desarrollo en las zonas rurales se ha visto obstaculizada por la multiplicidad de los componentes, la centralización de los procesos de decisión y la debilidad de las instituciones intermediarias (regionales y municipales). En muchos casos las políticas clientelistas y la falta de control administrativo han menoscabado los esfuerzos de descentralización. Los proyectos rurales han tropezado con el problema de que la ejecución sucesiva de sus diferentes componentes atenta contra la posibilidad de lograr los objetivos globales. Por ejemplo, en varios casos la construcción de carreteras rurales terminó antes de que comenzaran las actividades de asistencia técnica destinadas a regularizar la tenencia de las tierras, cosa que dio lugar al uso incontrolado de la tierra y a prácticas agrícolas insostenibles.

En general, los programas de desarrollo rural realizados en los años setenta y comienzos de los ochenta tuvieron un rendimiento menos que satisfactorio en cuanto a lograr sus objetivos físicos, y aún menos satisfactorio en cuanto a beneficiar a los grupos de in- 
gresos bajos (BID, 1989). Los problemas más citados son la escasa capacidad institucional, la insuficiente coordinación entre los organismos de ejecución y objetivos demasiado ambiciosos. La falta de información fidedigna ha dado lugar a proyecciones inexactas y a errores en los análisis de costo-beneficio y en la selección de objetivos y metas, cosa que ha provocado atrasos y costos excedidos. Sin embargo, muchos proyectos han tenido buenos resultados, particularmente los que han dado acceso a recursos productivos y servicios esenciales de los que anteriormente carecían los pobres de las zonas rurales, y han sido un buen ejemplo en materia de descentralización y participación comunitaria en el diseño y la ejecución de las actividades. Otra lección importante de los proyectos del pasado es la necesidad de crear un contexto político e institucional para la ejecución eficaz y descentralizada de los proyectos.

\section{V}

\section{Posibilidades de reducir la pobreza rural}

Aunque la pobreza rural se concentra principalmente entre los minifundistas con menor potencial agrícola y entre los habitantes rurales sin tierra, también son pobres algunos agricultores con potencial agrícola, principalmente porque no tienen suficiente acceso a la tecnología y el crédito, lo que les impide materializar el potencial de aumento de la productividad, o no tienen suficiente agua o seguridad en la propiedad de la tierra o no tienen suficiente acceso a los mercados para sus productos. La diferencia esencial entre los pobres del sector rural con mayor y menor potencial agrícola radica en la posibilidad que tienen los primeros de superar la situación de pobreza resolviendo estos problemas de comercialización, obteniendo los recursos financieros necesarios e invirtiendo, por ejemplo, en pequeñas obras de regadío o en nuevas tecnologías. ${ }^{3}$

Algunos de los medios más importantes para generar o elevar los ingresos entre los pobres en áreas rurales son los siguientes: desarrollo de la agricultura comercial, suministro de mayor acceso a la tierra, proyectos basados en los recursos naturales, promoción de microempresas e inversiones públicas y privadas en infraestructura. En aras de la sencillez, estas opciones se agruparán en tres categorías en el resto de este artículo: las que se basan en el crecimiento del sector

\footnotetext{
${ }^{3}$ El potencial agrícola se define como el acceso a los recursos (incluso terrenos de calidad adecuada y en cantidad suficiente) necesarios para generar los ingresos (o productos) que aseguren la supervivencia de la familia del agricultor y el desarrollo de la unidad de producción. Cuando no se da esta condición, el individuo afectado tiene menor potencial. La situación puede cambiar si se mejora el acceso a la tierra o se fomentan obras de riego en zonas de secano. Ambos grupos pueden coexistir dentro de una misma región o microrregión.
}

agrícola, las que apuntan a lograr el uso sostenible y la conservación de los recursos naturales, y las posibilidades que tiene la economía no agrícola en el ámbito rural.

\section{Opciones basadas en la agricultura}

Un plan de reducción de la pobreza rural que se base en la producción agrícola supondrá tanto abrir nuevas posibilidades de obtener recursos escasos como superar los obstáculos que se oponen a que los minifundistas logren aprovechar el pleno potencial de los recursos que controlan. Aunque los instrumentos propuestos puedan parecer tradicionales, los métodos aplicados para ponerlos en práctica no son los mismos del pasado, ya que operarán en lo posible por intermedio del mercado y del sector privado.

\section{a) Mercados de tierras eficaces}

La región posee todavía vastas extensiones de tierras de propiedad estatal, algunas de las cuales ofrecen un gran potencial tanto para la agricultura como para la silvicultura, sin que haya mucho peligro de agotar los recursos naturales. Además, existen grandes propiedades privadas que no están siendo explotadas productivamente. Muchos minifundistas y campesinos sin tierra tendrían la posibilidad de elevar sus ingresos si pudieran aprovechar esos recursos. Sin embargo, tras las tristes experiencias de los decenios de 1960 y 1970, la distribución de tierras entre los desposeídos no es una alternativa política viable. El énfasis que se da actualmente al uso más intensivo de la tierra, basado en nuevas tecnologías, debería mejorar las posibilidades de subsistencia de algunos campesinos sin tie- 
rra (aparceros) y abrirles nuevas posibilidades de empleo con los agricultores que tienen tierras, ya que la agricultura intensiva exige más mano de obra. Por lo tanto, deben promoverse los contratos de arrendamiento a plazo más largo, los acuerdos de aparcería y la distribución de las tierras fiscales para permitir el acceso de los pobres. Y estas actividades deben reforzarse con programas que ayuden a los agricultores a registrar la propiedad de las tierras que ocupan. Otros métodos pueden complementar estas iniciativas, como los programas de adquisición de tierras o la concesión de incentivos a los terratenientes para que vendan las propiedades mal explotadas, junto con programas que ayuden a los pequeños agricultores a comprar esas tierras. Del mismo modo pueden aplicarse sistemas tributarios progresivos sobre la propiedad que tasen la tierra según su potencial agrícola, a fin de desalentar la especulación y evitar grandes concentraciones de propiedad.

Según un estudio reciente sobre los mercados de tierras en la región (Echeverría, ed., 1998), la eficiencia de esos mercados puede mejorarse evitando las distorsiones en el uso de la tierra mediante el mantenimiento de un contexto macroeconómico, fiscal y sectorial neutro; consolidando los derechos de propiedad y rebajando los costos de transacción mediante la reforma de las políticas de asignación de tierras fiscales, y eliminando la reglamentación que limita la venta o arrendamiento de la tierra. De los muchos instrumentos para promover los mercados de tierras (reforma agraria y programas de colonización, impuestos sobre los bienes raíces y proyectos de titulación), los que pueden facilitar el acceso a la tierra para los minifundistas en particular son los programas de titulación y reforma agraria basados en el mercado. Al dejar la decisión sobre la selección de las tierras y la negociación de los precios en manos de los beneficiarios, el primer grupo de instrumentos podría eliminar varias causas de ineficiencia, aunque su costo sería sumamente elevado. Los programas actuales de titulación de tierras apuntan a crear registros de la propiedad rural que puedan actualizarse constantemente y a establecer sistemas para evaluar el impacto de la titulación. Estas actividades promueven innovaciones, como la participación de oficinas privadas en el proceso de regularización de títulos mediante licitaciones regionales financiadas por el erario; el establecimiento de nuevos reglamentos e incentivos financieros (o sanciones) para evitar la ulterior subdivisión de las propiedades o las ventas irregulares de tierras, y la vinculación de los proyectos de titulación con otros programas de apoyo agrario (transferencia de tecnología, programas de inversión y crédito).

\section{b) Mercados financieros}

Los mercados financieros rurales brindan a los habitantes del campo mayor acceso a los recursos financieros (crédito). Podrían desarrollarse estos mercados promoviendo y fortaleciendo instituciones financieras viables, estimulando el uso de métodos crediticios no tradicionales, estableciendo un marco regulador eficiente y derechos de propiedad bien definidos, creando nuevos mecanismos de garantía y estableciendo medios de cooperación entre las instituciones crediticias oficiales y oficiosas. Las zonas rurales carecen en gran medida de servicios financieros, sobre todo de instituciones oficiales de crédito. Por ello es necesario crear una nueva base institucional que permita a un número mayor de habitantes del campo conseguir préstamos y entrar en el circuito bancario. Específicamente, los programas de mercados financieros para el sector rural podrían incluir el apoyo a una legislación reguladora y a su aplicación, así como la reducción de los riesgos asociados a la producción y la comercialización. La reducción de los riesgos puede facilitarse promoviendo los seguros, los mercados a futuro, la cobertura de riesgo y el suministro adecuado de información. Hay varios aspectos esenciales para el desarrollo de los mercados financieros, como crear servicios crediticios no tradicionales adaptados a las condiciones del sector rural (préstamos colectivos en que los prestatarios responden de sus obligaciones conjunta y separadamente, intermediarios bancarios rurales y programas de fondos rotativos); introducir formas novedosas de atraer el ahorro que son de importancia decisiva para garantizar la viabilidad financiera de las instituciones, y establecer modos de cooperación entre las instituciones oficiales que poseen los recursos y las no oficiales que poseen la información necesaria para la selección y seguimiento de los clientes y la recuperación de los préstamos. Se dispone de diversos mecanismos para ofrecer servicios financieros alternativos como, por ejemplo, bancos de segunda instancia con fondos rotativos de apoyo al sector rural y la posibilidad de vincular los proyectos de apoyo a la microempresa con los servicios de crédito locales.

c) Desarrollo y transferencia de nuevas tecnologías Con contadas excepciones, los sistemas nacionales de investigación agrícola no han atribuido prioridad al desarrollo tecnológico en materia de agricultu- 
ra y de gestión de los recursos naturales ni a la transferencia de tecnología para elevar la productividad de los sistemas agrícolas del pequeño productor. Se necesita un mayor acento en la investigación aplicada, particularmente para adaptar las tecnologías disponibles a las condiciones específicas de los pequeños productores (falta de maquinaria, acceso limitado a los recursos financieros, temor a los riesgos). Gran parte del financiamiento y en algunos casos la ejecución de estas actividades, que son por definición locales y de interés público, seguirán siendo responsabilidad de las organizaciones públicas. Cada vez más proyectos de transferencia de tecnología son ejecutados por empresas privadas, organizaciones no gubernamentales y departamentos técnicos de asociaciones de comercio y financiados con fondos públicos en virtud de contratos adjudicados por licitación. Junto con fomentar el desarrollo y la transferencia de tecnología, es necesario capacitar a los pequeños agricultores, por ejemplo, en los métodos de administración financiera y gestión de los predios, en el registro de gastos y contabilidad financiera, en la reglamentación y los impuestos, así como en la explotación sostenible de los recursos naturales.

\section{d) Inversiones en pequeñas obras de regadío}

Rara vez los grandes proyectos de riego han beneficiado a los pequeños agricultores. En cambio, las inversiones en pequeñas obras (microrriego) y la introducción de mejoras tecnológicas (riego por goteo y miniaspersión) en pequeñas explotaciones agrícolas podrían tener resultados muy auspiciosos. La conversión de las tierras secas en explotaciones regadas tiene los mismos efectos en la productividad y en los ingresos de los predios que un aumento considerable de la superficie. Además, los proyectos de riego tienen un efecto favorable sobre el empleo rural.

\section{e) Promoción de nuevos métodos cooperativos de comercialización}

La economía rural debe hacer frente a altos costos de transacción en diversos mercados de productos básicos; de ahí que las zonas que tienen las mejores conexiones de mercado sean las más desarrolladas. Por consiguiente, es preciso hacer más hincapié en el desarrollo de los mercados rurales. La escasa capacidad negociadora de los pequeños agricultores en sus transacciones comerciales es un problema recurrente, sobre todo cuando los mercados se van haciendo cada vez más competitivos y exigen el funcionamiento de sistemas de información más complejos. Los pequeños productores pueden acceder al mercado de diversas maneras: merced a la creación de centros de información sobre los precios y al suministro de servicios de negociación (comprendido el asesoramiento técnico), al establecimiento de asociaciones para participar en iniciativas comerciales y a la difusión del uso de los contratos con la agroindustria.

\section{Iniciativas basadas en los recursos naturales}

Según las estimaciones, la población de América Latina aumentará a 670 millones para el año 2020, en tanto que la población rural se mantendrá constante, en términos absolutos, en unos 125 millones. Crecerá proporcionalmente la presión que ejerce la población urbana y rural sobre los recursos naturales de la región. Los campesinos pobres suelen no tener el equipo, los insumos y la tecnología necesarios para llevar a cabo obras de conservación o de reposición de los recursos naturales. Muchos no tienen asegurado el acceso a la tierra y los recursos que explotan, no pueden confiar en que recogerán los frutos de sus actividades de conservación y por lo tanto no tienen incentivo para proteger el agua, el suelo y la vegetación. Permitir el acceso a las tierras fiscales o no registradas sin un control social de su utilización es abrir el camino para el deterioro.

Al romperse el círculo de la pobreza y del deterioro de los recursos naturales puede originarse un círculo virtuoso en que la restauración de los recursos naturales contribuye a la reducción de la pobreza, pero para ello es preciso aplicar programas de apoyo que tengan esta finalidad concreta. Entre esos programas podrían figurar: un plan de incentivos a largo plazo para las familias rurales, que disponga el pago de una prima por unidad de tierra sustraída a la producción e incorporada en el programa de restauración de los recursos agotados (que continúe hasta que se haya logrado el objetivo de restauración); y un crédito especial tanto para invertir en la zona especial reservada para reforestación, explotación ganadera y forestal, restauración de la cubierta vegetal u otras prácticas de recuperación de suelos, como para transformar esa zona. Se trataría de programas a mediano plazo más bien que de proyectos de emergencia para la creación de empleos.

Como la agricultura no puede proporcionarle el sustento al minifundista cuyas tierras están agotadas, esas tierras y los demás recursos naturales podrían destinarse, por ejemplo, a la conservación de los ecosistemas y de la biodiversidad, a la preservación de 
los entornos naturales que por su belleza y recursos de vida silvestre podrían sustentar actividades recreativas $\mathrm{y}$ a otros usos generadores de ingresos como el ecoturismo asociado con la existencia de lagos, ríos, parques y otras reservas naturales. De este modo la demanda urbana de un mayor esfuerzo de restauración ambiental y de conservación podría encauzarse hacia actividades productivas que incrementarían los ingresos del sector rural desposeído.

Algunos elementos de la gestión sostenible de los recursos naturales que podrían aplicarse en los programas rurales son los siguientes: métodos de cultivos múltiples y de conservación, que aumenten el rendimiento sin agotar los recursos de suelos y aguas y a la vez mantengan o generen empleos; reformas legales para promover el uso racional y la gestión descentralizada de los recursos hídricos, garantizando el acceso equitativo a los recursos y el cumplimiento de la reglamentación pertinente; una administración de cuencas que establezca el debido equilibrio entre la necesidad de un desarrollo basado en la explotación sostenible de los recursos y la participación de la población rural; reformas de las estrategias y de la legislación para preservar los bosques naturales, su biodiversidad y su potencial para la producción de servicios ambientales, y para promover la reforestación con plantaciones comerciales a mediana escala; inclusión de las pesquerías de agua dulce en los planes y reglamentos para la gestión de los recursos hídricos, así como la capacitación y especialización en los servicios de apoyo a la acuicultura; y realce de las zonas naturales protegidas como factor primordial para el desarrollo rural y el uso sostenible de los recursos, prestando especial atención a la consolidación de sistemas nacionales de santuarios de la naturaleza, la participación de las comunidades rurales en la gestión y el aprovechamiento racional de los recursos, y la promoción del uso sostenible de los recursos biológicos, por ejemplo, en productos naturales alternativos.

\section{Actividades rurales no agrícolas}

Con suma frecuencia en la región se han empleado instrumentos de desarrollo agropecuario con el objeto de reducir la pobreza de los minifundistas que tienen escaso potencial agrícola. Como los minifundistas de menor potencial agrícola y los campesinos sin tierra representan una gran proporción de los habitantes rurales pobres, las actividades rurales no agrícolas son de mucha importancia para reducir la pobreza en la región. Una proporción creciente de la población rural trabaja fuera del sector agrícola (y un número creciente de trabajadores agrícolas vive en medios urbanos), por lo que reviste especial interés prestar mayor atención a los aspectos rurales no agrícolas y a los eslabonamientos entre lo urbano y lo rural, concibiendo la economía rural en su conjunto. Un estudio reciente (Reardon y Berdegué, 1999) muestra que en un promedio de 13 países el $47 \%$ del ingreso rural se originaba fuera del predio agrícola. Este resultado contradice la opinión generalizada que hace coincidir el ingreso rural con el ingreso del sector agrícola (ya sea por empleo asalariado o por explotación agrícola propia). Además, el empleo rural no agrícola, que representa el 30\% del total (Dirven, 1997), parece estar creciendo con rapidez mayor que el empleo agrícola. ${ }^{4}$

El hecho de que la migración contribuye a aliviar la pobreza rural es señal de que parte de la solución se encuentra al margen de la agricultura. Por lo tanto, es esencial mejorar la capacidad de la población rural para ingresar al mercado de trabajo, generar fuentes no agrícolas de empleo e ingreso y crear mejores condiciones para los que optan por trasladarse a un medio urbano. Aunque las actividades agrícolas seguirán siendo la fuente primaria de sustento para gran proporción de la población rural, hay que propender a la diversificación del empleo en las zonas rurales y al mejoramiento de la capacidad de trabajo de la población rural para que pueda competir en mejores condiciones en los mercados de trabajo urbanos que seguirán atrayendo a los integrantes suficientemente capacitados de la población rural. La ampliación de las oportunidades de empleo debe incluir el trabajo por cuenta propia en la agricultura, la pesca y la silvicultura, las pequeñas industrias y las artesanías, así como la creación de posibilidades de trabajo asalariado atrayendo a los empleadores del sector privado a las zonas rurales. Así, también debe prestarse mayor atención al fomento, de actividades productivas en el predio que, con ser agrícolas, no requieren mucha tierra (crianza de animales), así como a actividades no agrícolas que se desarrollan en el predio. En relación con las posibles actividades no agrícolas, se examinan a continuación las microem-

\footnotetext{
${ }^{4}$ La principal diferencia entre el empleo rural no agrícola y las proporciones de ingreso ( $30 \%$ frente a $47 \%$ ) podría explicarse (aparte la diversidad de fuentes y la existencia de datos no comparables) por la actividad plural, es decir, que los ingresos derivan de varias fuentes sectoriales en el año para cada individuo y comparando a distintos individuos de una misma familia. La proporción de hogares rurales que no se especializan sectorialmente y que por lo tanto son "pluriactivos" puede ser bastante alta (40\% en Nicaragua, según Reardon y Berdegué,1999), lo que no suele tomarse en cuenta en un censo que sólo pregunta sobre la "ocupación primaria".
} 
presas, la capacitación y la inversión en infraestructura rural.

\section{a) Desarrollo de microempresas rurales}

Más de la mitad de los empleos de la región corresponden a empresas con menos de 10 trabajadores, y el $50 \%$ de ellas son empresas familiares. El crecimiento más acelerado se da en las microempresas dirigidas por mujeres. Es esencial para el desarrollo de tales microempresas crear las condiciones que les permitan ser competitivas e independientes de la ayuda externa, estableciendo un marco regulador y políticas favorables y mejorando su acceso a los servicios financieros y comerciales. Además, los esfuerzos de descentralización y mejora de los servicios financieros rurales contribuirán al desarrollo de pequeñas empresas y microempresas en las zonas rurales, especialmente allí donde pueda mejorarse el acceso a los préstamos de poco monto.

Algunas microempresas se adaptan especialmente bien a la economía rural: agroindustrias, talleres de reparación de maquinaria, talleres y tiendas de artesanía, pesca comercial y deportiva, fábricas de muebles y productos de madera, y las relacionadas con el turismo (pequeños restoranes y hoteles, instalaciones termales y sitios de interés cultural). El sector rural ofrece grandes posibilidades para fabricar, a partir de materiales naturales, diversos artículos y artesanías, como productos de madera, hierbas medicinales, fibras, frutas y nueces, flores, piedras semipreciosas, todos ellos materiales que favorecen el uso sostenible de estos recursos.

\section{b) Capacitación profesional para mejorar las opor- tunidades de empleo y los ingresos}

Como la educación primaria y la secundaria se consideran determinantes tanto del tipo de empleo como de los ingresos que pueden obtenerse, la demanda de estos servicios es sumamente alta entre la población rural. Se trata una vez más de una esfera decisiva en la que los gobiernos deben concentrarse para garantizar un nivel básico de educación formal en las zonas rurales. Además, en muchos de los países de la región la capacitación profesional disponible no ha tomado en cuenta la demanda comercial de especialidades y técnicas. Por ejemplo, en muchos casos el número de programas de capacitación agrícola destinados a los jóvenes supera de lejos el número de posibles empleos en el sector. De lo que se trata es de mejorar las oportunidades tanto para los hombres como para las mujeres entre los pobres de las zonas rurales, capacitándolos en técnicas que vayan aparejadas con la demanda de mano de obra en las zonas urbanas y en las rurales e incluso preparándolos para el trabajo por cuenta propia. Para ello pueden resultar muy eficientes los programas de capacitación profesional (como los programas cooperativos de estudio y trabajo) realizados en conjunto con empresas privadas.

\section{c) Atraer inversiones públicas y privadas en infra- estructura}

Las inversiones públicas y privadas con incentivos fiscales y otros incentivos financieros (como la creación de parques industriales con incentivos públicos para que la industria privada se instale en las zonas rurales), la distribución de tierras y los proyectos de reforestación y regadío, la electrificación rural, el turismo y otros planes concebidos para canalizar las inversiones hacia las zonas rurales pueden tener un gran efecto de generación de empleo para los pobres de las zonas rurales.

Es especialmente importante corregir los desequilibrios regionales (entre las zonas urbanas y las rurales y también dentro de las zonas rurales) y promover regiones que tengan, por ejemplo, un buen potencial de recursos naturales. En esta esfera, la inversión en caminos y electricidad, es un medio eficaz para atraer inversiones externas hacia las zonas rurales marginalizadas y al mismo tiempo permitir que lleguen servicios a los grupos de menos ingresos y se reduzcan los costos de las transacciones que supone la comercialización de la producción rural. La falta de caminos transitables todo el año es un grave obstáculo a los esfuerzos por atraer las inversiones del sector privado a la industria rural, la minería y el turismo. La inversión en la infraestructura vial de las zonas rurales, además de favorecer el empleo local en la industria de la construcción, mejora el acceso a los servicios sociales, reduce los costos de las transacciones comerciales, abre nuevos mercados de trabajo y genera externalidades que benefician a los pobres de las zonas rurales. 


\section{VI}

\section{Conclusiones}

A pesar de un período de crecimiento económico considerable en la mayoría de los países de América Latina y el Caribe en los años noventa, la pobreza rural no ha disminuido de manera apreciable. De 1994 a 1997 de hecho aumentó el número de habitantes en situación de pobreza. Además, la pobreza urbana también se ve alimentada por la migración de los pobres del campo a la ciudad. El paisaje rural de América Latina y el Caribe es muy variado y se vincula cada vez más al medio urbano, albergando a una población relativamente escasa. Existen también grandes variaciones en cuanto a la naturaleza y magnitud de la pobreza en las zonas rurales. Aunque la agricultura sigue siendo la principal fuente de empleo, están adquiriendo creciente importancia las actividades rurales no agrícolas, que son bastante interdependientes con los centros urbanos. Dado que los elementos en juego son más generales, interconectados y multisectoriales, los problemas económicos y sociales de las zonas rurales deben plantearse sobre una base territorial más que exclusivamente sectorial.

Además de las diferencias de cultura, geografía rural y dotación de recursos naturales, así como los aspectos institucionales y políticos que caracterizan a los países de la región, hay también numerosas diferencias regionales y subregionales dentro de cada país. Esta diversidad de condiciones rurales tiene gran relevancia a la hora de elegir criterios de desarrollo rural de la economía. Para los organismos multilaterales, apunta a la necesidad de mantener un diálogo abierto y continuo con cada país para definir y conformar proyectos destinados a solucionar problemas específicos. Para cada país, significa un fuerte compromiso de descentralización a fin de dar cabida a la diversidad y estimular a los distintos agentes a expresar sus opiniones. Para la región o subregión en que se desarrollarán las actividades, impone una gran flexibilidad a fin de velar por que, en su aplicación, los planes concuerden con las posibilidades e intereses de la población. Y por último, en el plano de la comunidad, significa que el criterio elegido debe estimular la participación de todos los beneficiarios y entidades cooperantes ( organizaciones no gubernamentales, grupos comunitarios, dependencias gubernamentales, municipalidades) en el diseño, ejecución y evaluación de las iniciativas rurales.
Prestar especial atención al desarrollo de áreas rurales (para reducir el sesgo a favor de las zonas urbanas) podría compensar el déficit acumulado en los gastos en infraestructura rural. Para ello será preciso compensar las deficiencias en los programas de capacitación, establecer una infraestructura rural básica, incentivar a la inversión privada, y dar acceso a las personas con menos recursos del sector rural a los servicios y mercados disponibles para la población urbana. Es importante que estos programas sean transparentes y orientados hacia los segmentos más pobres, evitando una transferencia general de recursos. Por otro lado, para vincular el crecimiento económico con la reducción de la pobreza en las zonas rurales habría que contar con mecanismos que velen por que los beneficios del crecimiento económico lleguen a quienes no tienen peso suficiente para ganar acceso a ellos, sobre todo los grupos más vulnerables como los jóvenes, las mujeres y las comunidades indígenas.

Hay indicios de que las actividades rurales no agrícolas constituyen una fuente cada vez más importante de ingresos para los hogares rurales de todo tipo, incluso los de los campesinos sin tierra y los residentes en pueblos rurales. Pese a esta tendencia, la mayoría de las iniciativas encaminadas a aliviar la pobreza rural no ha aprovechado plenamente las posibilidades de empleo para los asalariados y los trabajadores por cuenta propia generadas en actividades como el comercio, la manufactura y otros servicios. Las relaciones de la economía rural no agrícola con los mercados de insumos y productos y de servicios son variables claves en la definición de las políticas rurales de la región, sobre todo en lo que toca al desarrollo de microempresas y pequeñas empresas, como las pequeñas industrias artesanales, las elaboradoras de productos agrícolas (de importancia creciente en la economía rural), los servicios relacionados con el sector agrícola, y otras actividades (turismo, por ejemplo).

De igual utilidad en cuanto a proporcionar empleo para los pobres en áreas rurales, así como para fines ambientales, son otras iniciativas de inversión vinculadas a los recursos naturales, como la reforestación, la conservación de suelos, el manejo de cuencas hidrográficas y la creación y administración de parques 
nacionales y reservas naturales. Las iniciativas basadas en la agricultura constituyen una tercera opción importante para reducir la pobreza en la región; entre ellas figuran el desarrollo de los mercados de tierras y aguas y del mercado financiero, las inversiones en nuevas tecnologías y riego y también la promoción de nuevos métodos de comercialización.

De las distintas posibilidades para reducir la pobreza rural en la región, hay cinco que son cruciales, a saber: i) la migración a las zonas urbanas con mayor demanda de empleo, que ha sido la solución tradicional, y podría mejorarse dando capacitación a los migrantes para que puedan obtener mejores empleos de base urbana; ii) las redes de seguridad social para la población que no tiene una opción productiva rural o agrícola y que no puede migrar por razones de salud, edad, etc.; iii) las actividades rurales no agrícolas; iv) los proyectos en la recuperación y conserva- ción de los recursos naturales, y v) las actividades con base en el agro para los pequeños agricultores con potencial agrícola. En la realidad, estas posibilidades se complementan; así, por ejemplo, un pequeño agricultor con escaso potencial agrícola puede desarrollar varias actividades económicas fuera de su predio para complementar su ingreso, mientras que los habitantes rurales sin acceso a la tierra también pueden diversificar sus ingresos trabajando en el campo. Dada la situación crítica de pobreza en las zonas rurales, la migración y la seguridad social probablemente seguirán siendo opciones importantes. Se requieren significativas inversiones en un desarrollo agrícola sostenible y en actividades económicas rurales no agrícolas para lograr el crecimiento económico y la reducción de la pobreza rural.

(Traducción del inglés)

Bibliografía

BID (Banco Interamericano de Desarrollo) (1989): Interim Assessment of the Effects of Bank Projects on Rural Development: A Summary of Ex-Post Evaluations, OEO PPR-40/89, Washington, D.C.

(2000): Estrategia para el desarrollo agroalimentario en América Latina y el Caribe, SDS RUR-102, Washington D.C.

Binswanger, H.P. y K. Deininger (1997): Explaining agricultural and agrarian policies in developing countries, Journal of Economic Literature, vol. 35, $\mathrm{N}^{\circ}$ 4, Nashville, Tennessee, American Economic Association.

CEPAL (Comisión Económica para América Latina y el Caribe) (1999): Panorama social de América Latina, 1998, LC/G.2050-P, Santiago de Chile.

Dirven, M. (1997): El empleo agrícola en América Latina y el Caribe: pasado reciente y perspectivas, Desarrollo productivo, $\mathrm{N}^{\circ}$ 43, LC/G.1961, Santiago de Chile, CEPAL.

Echeverría, R.G. (1998): Strategic Elements for the Reduction of Rural Poverty in Latin America and the Caribbean, Technical paper, $\mathrm{N}^{\circ}$ ENV-112, Washington, D.C., Banco Interamerica- no de Desarrollo (BID), Departamento de Desarrollo Sostenible, División de Medio Ambiente.

Echeverría, R.G., ed. (1998): Perspectivas sobre mercados de tierras rurales en América Latina, Washington, D.C., BID.

FAO (Organización de las Naciones Unidas para la Agricultura y la Alimentación) (1988): Potencialidades de desarrollo agrícola y rural en América Latina y el Caribe, Roma.

López, R. y A. Valdés (1997): Rural Poverty in Latin America: Analytics, New Empirical Evidence, and Policy, Washington, D.C., Banco Mundial, Departamento Técnico.

Piñeiro, M., R. Martínez Nogueira, G. Trigo, F. Torres, E. Manciana y R. G. Echeverría (1999): La institucionalidad en el sector agropecuario de América Latina. Evaluación y propuestas para una reforma institucional, SDS RUR-101, Washington D.C., Banco Interamericano de Desarrollo.

Reardon, T. y J. Berdegué (1999): Rural non-farm employment and incomes in Latin America, trabajo presentado al IDB/ECLAC/ FAO/RIMISP Seminar on Rural Non-Farm Employment, Santiago de Chile, 6 al 8 de septiembre. 